\title{
A Síndrome dos Ovários Policísticos Pode Interferir nos Resultados da Fertilização In Vitro?
}

Can Polycystic Ovary Syndrome Interfere with the Outcome of In Vitro Fertilization?

Rosana Maria dos Reis, Alexandre Gonçalves De Ângelo, Gustavo Salata Romão

Laura Ferreira Santana, Marcos Dias de Moura, Rui Alberto Ferriani

\section{RESUM0}

Objetivo: avaliar os resultados da superindução de ovulação seguida de fertilização in vitro (FIV) em pacientes com síndrome dos ovários policísticos (SOP), comparado-as a mulheres com ovários normais.

Métodos: estudo retrospectivo, controlado, no qual foram incluidas 36 mulheres com SOP (grupo SOP) e 44 mulheres que apresentavam infertilidade por fator masculino leve (grupo controle), submetidas à FIV no periodo de 1997 a 2003. A idade variou de 18 a 36 anos. A superindução da ovulação foi realizada com hormônio folículo-estimulante recombinante $e$ agonista do hormônio liberador de gonadotrofinas. As variáveis analisadas foram os folículos com diâmetros médios entre $14 \mathrm{~mm}$ e $17 \mathrm{~mm}$ e foliculos com diâmetros maiores ou iguais a $18 \mathrm{~mm}$ no dia da administração de gonadotrofina coriônica humana, porcentagem de folículos $\geq 18 \mathrm{~mm}$, número de oócitos captados, taxa de fertilização, taxa de clivagem, incidência de sindrome de hiperestimulação ovariana (SHO), taxa de gravidez clínica e taxa de abortamento. Estas variáveis foram analisadas pelo testes t não pareado, exato de Fisher e Mann-Whitney. Resultados: o grupo SOP apresentou maior número de folículos recrutados, a maioria com diâmetro entre 14 e $17 \mathrm{~mm}$, quando comparado ao grupo controle (64,8 versus 53,9\%), menor taxa de fertilização $(59,4$ versus $79,6 \%)$ e maior incidencia de $\mathrm{SHO}(38,9$ versus $9,1 \%)(p<0,05)$. O número de oócitos captados, as taxas de clivagem, de gestação por transferência de embriões, de aborto e de recém-nascido vivo não diferiram entre os grupos.

Conclusão: o sucesso da FIV está comprometido em mulheres com SOP por apresentarem recrutamento de maior número de folículos com diâmetros reduzidos, taxa reduzida de fertilização e elevada taxa de SHO.

PALAVRAS-CHAVE: Síndrome dos ovários policísticos. Fertilização in vitro. Indução da ovulação. Taxa de fertilização. Síndrome de hiperestimulação ovariana.

\section{Introdução}

A síndrome dos ovários policísticos (SOP) é caracterizada pela pluralidade de seus diversos

Departamento de Ginecologia e Obstetrícia da Faculdade de Medicina de Ribeirão Preto da Universidade de São Paulo Correspondência:

Rosana Maria dos Reis

Departamento de Ginecologia e Obstetrícia da Faculdade de Medicina de Ribeirão Preto da Universidade de São Paulo Av. Bandeirantes, 3900

14049-900 - Ribeirão Preto - SP

Fone: (16) 602-2926/Fax: (16) 633-0946

e-mail: romareis@fmrp.usp.br achados clínicos e laboratoriais ${ }^{1}$. De acordo com consenso recente, o diagnóstico da SOP é realizado pela presença de dois dos seguintes parâmetros: irregularidade menstrual, hiperandrogenismo clínico e/ou laboratorial e morfologia de ovários policísticos rodeando um estroma denso à ultrasonografia ${ }^{2}$.

A SOP é causa freqüente de infertilidade anovulatória e hirsutismo ${ }^{3,4}$. Além disso, o hiperandrogenismo ovariano pode ser um dos principais fatores responsáveis pela anovulação persistente $^{5}$ e, juntamente com a hiperinsulinemia freqüentemente associada a SOP, pode contribuir para a infertilidade nestas pacientes. 
A restauração da fertilidade normal em pacientes com SOP é possivel com o uso de diferentes protocolos de indução de ovulação, utilizandose drogas de fácil manuseio, como o citrato de clomifeno. Possiveis problemas relacionados ao seu uso incluem a falta de desenvolvimento de um folículo pré-ovulatório, assim como o desenvolvimento de sindrome de hiperestimulação ovariana (SHO), complicação freqüentemente associada a qualquer tipo de estimulação ovariana de pacientes com $\mathrm{SOP}^{6}$. O tratamento de pacientes que não respondem ao uso de citrato de clomifeno é complexo. A indução de ovulação com gonadotrofinas exógenas está associada a mais baixas taxas de ovulação e gravidez do que as obtidas em mulheres com hipogonadotrofismo, e a aumento da chance de apresentar múltiplo desenvolvimento folicular e maior risco de SHO e aborto $^{7-9}$.

A fertilização in vitro (FIV) demonstrou ser tratamento eficaz para mulheres com $\mathrm{SOP}^{10,11}$ refratárias ao tratamento com indutores da ovulação apenas ${ }^{12}$. A evolução do tratamento com superovulação e FIV em mulheres com SOP parece ser similar à de mulheres com ovários normais, no que diz respeito a taxas de gravide ${ }^{13}$. Entretanto, mulheres com SOP têm maior risco de desenvolver SHO após superindução de ovulação e FIV $^{11}$, altas taxas de cancelamento de ciclos, maior número de oócitos coletados, porém mais baixas taxas de fertilização e de clivagem ${ }^{12}$, e menor taxa de implantação ${ }^{14}$.

Tendo em vista possivel pior desempenho de pacientes com SOP quando submetidas à superindução da ovulação e FIV, comparamos neste estudo os resultados obtidos em um programa de FIV em pacientes com SOP com aqueles obtidos por mulheres com ovários normais.

\section{Pacientes e Métodos}

Realizamos estudo retrospectivo e controlado incluindo mulheres submetidas à FIV no Setor de Reprodução Humana do Hospital das Clínicas de Ribeirão Preto da Universidade de São Paulo (HCFMRP-USP) no período de 1997 a 2003. O grupo de estudo foi constituído de 36 pacientes com SOP (grupo SOP), sem outro fator de infertilidade. $\mathrm{O}$ diagnóstico foi feito pela presença de oligo ou amenorréia e hirsutismo clínico (índice de Ferriman maior que 8) ${ }^{15}$ ou laboratorial (niveis plasmáticos de testosterona superior a $80 \mathrm{ng} / \mathrm{mL}$ ) e ultra-sonografia com presença de ovários policísticos bilateralmente, com 12 ou mais folículos com diâmetro de 2 a 9 mm rodeando den- so estroma, ou volume ovariano aumentado (maior que $10 \mathrm{~cm}^{3}$ ). O grupo controle foi constituido por 44 mulheres que apresentavam infertilidade por fator masculino leve exclusivo, caracterizado por concentração de espermatozóides progressivos maior que 10 milhões/mL no recuperado, após a capacitação espermática, e mais de $8 \%$ de espermatozóides normais.

Todas as pacientes selecionadas haviam sido submetidas ao protocolo básico de investigação de infertilidade do HCFMRP-USP antes de iniciar o tratamento de indução da ovulação. Essa investigação consiste em exame físico ginecológico, ultra-sonografia transvaginal, histerossalpingografia, histeroscopia, laparoscopia, dosagem de hormônio folículo-estimulante (FSH) e PRL no $3^{\circ}$ dia do ciclo menstrual e avaliação de espermograma do parceiro.

Foram critérios de inclusão a idade entre 18 e 36 anos. Foram critérios de exclusão a presença de outro fator de infertilidade, como endometriose e fator tubáreo, e má resposta à indução de ovulação (menos de dois folículos com mais de 17 mm de diâmetro).

As pacientes foram submetidas a bloqueio hipofisário com agonista do hormônio liberador das gonadotrofinas (GnRHa), iniciando na metade da fase lútea do ciclo anterior, e à hiperestimulação ovariana controlada com FSH recombinante (FSHr). A injeção de 5000 a 10000 unidades (UI) de gonadotrofina coriônica humana (hCG) foi efetuada quando apresentassem ao ultra-som pelo menos dois folículos com mais de $18 \mathrm{~mm}$ de diâmetro médio. A fase lútea foi complementada com progesterona natural.

Os espermatozóides presentes no ejaculado obtido por masturbação foram preparados pelo gradiente descontínuo de PureSperm (PureSperm Nidacon - International $\mathrm{AB}$ ), utilizando-se duas concentrações de 45 e 90\%. O critério de normalidade utilizado foi o estabelecido pela Organização Mundial de Saúde (WHO, 1994) ${ }^{16}$, com exceção da morfologia, que foi avaliada pelos critérios de Kruger et al. (1986) ${ }^{17}$.

O critério diagnóstico para obesidade foi o índice de massa corporal (IMC), calculado por peso $(\mathrm{kg}) /$ altura $\left(\mathrm{m}^{2}\right)$.

Os critérios empregados para a comparação entre os grupos foram: número de folículos com diâmetros médios entre 14 e $17 \mathrm{~mm}$, número de folículos com diâmetros maiores ou iguais a 18 $\mathrm{mm}$ no dia da administração de hCG, proporção de folículos maiores ou iguais a $18 \mathrm{~mm}$ em relação aos folículos entre 14 e $17 \mathrm{~mm}$, número de oócitos captados, taxa de fertilização (razão entre os oócitos 
fertilizados pelos oócitos inseminados), taxa de clivagem (razão entre o número total de embriões pelos oócitos fertilizados), incidência de SHO (classificada em leve, moderada ou grave ${ }^{7}$ ) e taxa de gravidez clínica e de abortamento.

Os dados obtidos das pacientes foram inseridos em banco de dados do programa Microsoft Access $2002^{\circ}$. A análise estatística foi realizada com auxílio do software GraphPad Prism ${ }^{\circledR}$ versão 3.00. Foram utilizados o teste $t$ não pareado, para análise de variáveis numéricas paramétricas (número total de folículos recrutados), e o teste exato de Fisher para análise das porcentagens (porcentagem de folículos recrutados entre 14 e $17 \mathrm{~mm}$ e de folículos $\geq 18 \mathrm{~mm}$, taxa de fertilização, de clivagem, de abortamento, de gestação, de recémnascidos vivo e de $\mathrm{SHO}$ ).

O protocolo desta pesquisa foi analisado e teve aprovação do Comitê de Ética em Pesquisa do
Hospital das Clínicas da Faculdade de Medicina de Ribeirão Preto da Universidade de São Paulo.

\section{Resultados}

A média de idade das pacientes estudadas foi semelhante entre os grupos, sendo 30,5 anos (variação de 24 a 36 anos) no grupo SOP e 32,0 (variação de 23 a 35 anos) no grupo controle (Tabela 1). Entre as pacientes com SOP, a irregularidade menstrual estava presente em $86,1 \%$, hirsutismo em $44,4 \%$ e ovários policísticos e/ou aumento do volume ovariano ao exame de ultrasom transvaginal em $75 \%$ delas. As pacientes com SOP apresentaram maior IMC comparadas ao grupo controle $\left(27,9\right.$ vs $23,8 \mathrm{~kg} / \mathrm{m}^{2}$, respectivamente) $(\mathrm{p}=0,0002)$ (Tabela 1$)$.

Tabela 1 - Principais características clínicas e parâmetros da indução da ovulação das 80 pacientes incluídas no estudo.

\begin{tabular}{lccc}
\hline & SOP $(\mathbf{n}=\mathbf{3 6})$ & Controles $(\mathbf{n}=\mathbf{4 4})$ & $\mathbf{p}$ \\
\hline Idade (anos) & $30,5 \pm 3,3$ & $32,0 \pm 3,6$ & 0,0619 \\
IMC $\left(\mathrm{kg} / \mathrm{m}^{2}\right)$ & $27,9 \pm 5,9$ & $23,8 \pm 3,2$ & 0,0002 \\
Espessura do endométrio (mm) & $11,0 \pm 1,8$ & $11,6 \pm 3,0$ & 0,3336 \\
Dias de indução & $9,8 \pm 2,1$ & $9,3 \pm 1,5$ & 0,2365 \\
Ul de FSHr utilizadas & $1717,0 \pm 604,2$ & $2593,1 \pm 713,2$ & $<0,0001$ \\
\hline
\end{tabular}

Teste $t$ não pareado. SOP = síndrome dos ovários policísticos; IMC = índice de massa corporal; FSHr = hormônio folículo-estimulante recombinante.

Não houve diferença no número de dias de estimulação ovariana ( $\mathrm{SOP}=9,8 v$ s controle $=9,3$ dias), mas as pacientes com SOP necessitaram de maior número de unidades (UI) de FSHr (1.717,0 vs 2.593,1 UI) (Tabela 1), assim como nelas o número de oócitos obtidos foi menor $(11,6$ vs 8,8) (Tabela 2). Quando comparado ao grupo controle, o grupo SOP apresentou maior número de folículos recrutados $(15,3$ vs 9,2$)$, sendo a maioria com diâmetro entre 14 e 17 mm ( 64 vs 53,9\%), menor taxa de fertilização $(59,4$ vs $79,5 \%)$ e maior incidência de $\mathrm{SHO}(38,8$ vs $9 \%)$. As taxas de clivagem $(76,9$ vs $75,1 \%)$, de abortamento $(46,6$ vs $28,5 \%)$, de gestação $(47,2$ vs $54,5 \%)$ e de recém-nascido vivo $(22,2$ vs 34\%) foram similares (Tabela 2).

Tabela 2 - Resultados de indução de ovulação e fertilização in vitro comparando-se as mulheres com síndrome dos ovários policísticos e do grupo controle.

\begin{tabular}{|c|c|c|c|}
\hline & SOP $(n=36)$ & Controle $(n=44)$ & $\mathrm{p}$ \\
\hline Folículos recrutados $\geq 18 \mathrm{~mm}$ ( $\mathrm{n}^{\circ}$ médio e $\left.\%\right)$ & $5,4(35,2 \%)$ & $4,2(46,1 \%)$ & $0,0007^{*}$ \\
\hline Número médio de oócitos captados & 11,6 & 8,8 & $0,0505^{\star x}$ \\
\hline Taxa de fertilização (\%) & 59,4 & 79,5 & $<0,0001^{*}$ \\
\hline Taxa de gestação (\%) & 47,2 & 54,5 & $0,6534^{*}$ \\
\hline Taxa de recém-nascido vivo (\%) & 22,2 & 34,0 & $0,3224^{*}$ \\
\hline $\mathrm{SHO}(\%)$ & 38,8 & 9,0 & $0,0025^{*}$ \\
\hline
\end{tabular}

Teste exato de Fisher $\left(^{*}\right) ; t$ não pareado $\left(^{* *}\right)$.

$\mathrm{SHO}=$ síndrome de hiperestimulação ovariana. 
A SHO, além de mais freqüente, foi mais intensa nas pacientes com SOP: obtivemos 35,7\% de SHO leve $(n=5), 57 \%$ de moderada $(n=8)$ e $7,1 \%$ de grave $(n=1)$, ao passo que no grupo controle $75 \%$ foi da forma leve $(n=3)$ e $25 \%$ moderada $(n=1)(T a-$ bela 3). A taxa de gravidez clínica entre as mulheres que apresentavam SHO e SOP não foi diferente daquelas que não apresentavam SHO e SOP, resultando em gestações em metade dos casos $(\mathrm{n}=7)$. Nas mulheres do grupo controle com SHO, $75 \%(n=3)$ apresentaram gestação clínica.

Tabela 3 - Porcentagem de pacientes que desenvolveram SHO nos grupos com síndrome dos ovários policísticos e controle.

\begin{tabular}{lcc}
\hline SHO & SOP $(\mathbf{n}=\mathbf{3 6})$ & Controles $(\mathbf{n}=\mathbf{4 4})$ \\
\hline Leve & $5(35,71 \%)$ & $3(75,0 \%)$ \\
Moderada & $8(57,14 \%)$ & $1(25,0 \%)$ \\
Grave & $1(7,15 \%)$ & - \\
Total & 14 & 4 \\
\hline
\end{tabular}

\section{Discussão}

A SOP é doença multifatorial, que ocasiona ciclos anovulatórios e infertilidade, além de hirsutismo, obesidade, hiperinsulinemia e diabete $^{1,8,9}$. A abordagem terapêutica é bastante complexa, daí a grande quantidade de estudos envolvendo os múltiplos aspectos das respostas a tratamentos, especialmente aqueles ligados à indução de ovulação.

Comparamos em nosso estudo a resposta de pacientes com SOP submetidas a superindução de ovulação e FIV com um grupo controle cujo único fator de infertilidade era a presença de um fator masculino leve, no sentido de termos pacientes controles com ovários normais. Tal fato pode levantar a discussão de que os resultados ligados à fertilização sofressem influência negativa de defeito dos gametas masculinos deste grupo. Entretanto, escolhemos grupo de pacientes com fator masculino leve, com concentrações de espermatozóides progressivos superior a 10 milhões/ mL após capacitação espermática, com morfologia normal (mais de 8\%), utilizando os critérios estritos de Kruger, parâmetros estes que não interferem na fecundação oocitária ${ }^{18}$.

A FIV é procedimento indicado para pacientes com SOP quando refratárias a processos de indução de ovulação não associada a procedimentos invasivos, com boa relação custo-benefício ${ }^{19}$. Os esquemas de indução de ovulação para pacientes com SOP costumam associar um bloqueio hipofisário com GnRHa, a fim de prevenir pico prematuro de LH endógeno em resposta aos níveis elevados de estradiol que acompanham o crescimento multifolicular destas pacientes. Os picos inadvertidos de LH podem promover ovulações prematuras, além de piorar substancialmente a qualidade dos oócitos. O uso do bloqueio hipofisário pode também propiciar maior sincronismo do crescimento folicular e maior conveniência quanto ao momento da captação de oócitos e transferência embrionária.

Alguns estudos mostram que mulheres com SOP associado a uma fase folicular com concentração sérica aumentada de LH têm risco aumentado para aborto espontâneo ${ }^{20,21}$. Níveis elevados de LH de pacientes com SOP podem afetar adversamente a maturidade oocitária durante a indução da ovulação e conseqüentemente produzir oócitos não viáveis para fertilizar ${ }^{21,22}$. Para isso, o uso de GnRHa tem sido utilizado para supressão dos níveis de $\mathrm{LH}$ e, desta maneira, melhorar os efeitos deletérios produzidos por ele durante o tratamento de infertilidade ${ }^{23}$, com melhora nas taxas de gravidez e redução nas taxas de aborto ${ }^{20}$.

Além do nível elevado de LH sérico, outro fator capaz de causar elevadas taxas de abortamento é o peso corpóreo aumentado destas pacientes. Wang et al. ${ }^{24}$ mostraram que mulheres obesas com SOP apresentaram riscos elevados para aborto espontâneo comparado com controle ( 25 versus $18 \%$, respectivamente). Em nosso estudo as mulheres com SOP e com IMC elevado $\left(>30 \mathrm{~kg} / \mathrm{m}^{2}\right)$ mostraram maior freqüência de abortamento espontâneo quando comparadas com o grupo controle, embora não tenha havido diferença significativa entre os grupos.

Tem sido observada alta taxa de cancelamento de transferência embrionária em ciclos de reprodução assistida em pacientes com $\mathrm{SOP}^{25}$, devido principalmente a SHO. Esta pode se transformar em grave complicação e adquirir formas ameaçadoras, aumentando a morbidade e mortalidade destas pacientes. Sua gravidade depende do grau de evolução destes sintomas em cada pacien$\mathrm{te}^{26}$. A incidência da SHO severa tem sido estimada em 0,2 a $1 \%$ de todos os ciclos de reprodução assistida $^{26}$ e está associada com mortalidade entre 1:45.000-1:50.000 por mulheres inférteis recebendo gonadotrofinas ${ }^{27}$.

$\mathrm{Na} \mathrm{SOP}$, as mulheres apresentam resposta excessiva à estimulação ovariana devido ao fato de os ovários policísticos apresentarem vários folículos parcialmente desenvolvidos que estariam prontos para a estimulação, propiciando típica resposta multifolicular. Os folículos ovarianos seriam altamente sensiveis ao $\mathrm{FSH}$, o que fa- 
vorece aumento dos receptores devido à alta concentração local de estrógenos e, como resultado, haveria desenvolvimento multifolicular associado com alta concentração de estrógenos circulante ${ }^{27}$.

A alta sensibilidade destas pacientes em face dos estimuladores ovarianos justifica mudanças na abordagem terapêutica. Em nosso estudo, doses reduzidas de gonadotrofinas foram utilizadas no grupo de pacientes com SOP quando comparado ao grupo controle. Relatos da literatura mostram que mulheres com SOP em tratamento com FIV são capazes de recrutarem quantidade maior de folículos e também de oócitos após estimulação ovariana, mesmo com doses reduzidas de $\mathrm{FSHr}^{11,12,28}$. Outras particularidades dos resultados da FIV em pacientes com SOP são oócitos de baixa qualidade ${ }^{29}$, pouca maturidade ${ }^{30}$ e conseqüentemente com maior dificuldade de fertilização oocitária ${ }^{11}$.

No nosso estudo, as pacientes com SOP apresentaram maior quantidade de folículos recrutados com diâmetros menores ( $\geq 14 \mathrm{~mm}$ e $\leq 17 \mathrm{~mm}$ ) quando comparadas ao grupo controle. No entanto, a taxa de fertilização foi mais baixa e não se observaram alterações nas taxas de clivagem, gravidez e de recém-nascido vivo. Estes dados confirmam alguns estudos prévios ${ }^{12,31}$. Porém, o estudo de Engmann et al. ${ }^{32}$ mostrou que mulheres com achados ultra-sonográficos de ovários policísticos, mas sem sintomas clínicos associados à SOP, submetidas à reprodução assistida, não apresentaram alterações nas taxas de fertilização $(59,4$ versus $60,2)$ e de implantação $(18,6$ versus 11,7$)$ quando comparadas a mulheres com ovários normais ao ultra-som.

As taxas de gestação e de clivagem em mulheres com SOP são dados de controvérsia na literatura. Os estudos divergem, mostrando em um subgrupo de pacientes com SOP obesas e com resistência à insulina baixa taxa de implantação ${ }^{14}$, ao passo que em outros a taxa de gestação não apresenta diferença quando comparada com mulheres com ovários normais ${ }^{11}$ ou com infertilidade devida a fator tubáreo ${ }^{12,29}$. A taxa de clivagem mostrou estar reduzida em alguns relatos da literatura em relação a mulheres com infertilidade tubárea $^{12}$, sendo que em outros não apresentou diferença quando comparada a mulheres com ovários normais ${ }^{11}$.

A SHO é achado freqüente em mulheres com SOP que são submetidas a estimulação ovariana controlada em ciclos de FIV ${ }^{14,29,33}$. Esse fenômeno é mediado por substâncias vasoativas que provocam alterações no compartimento vascular. O estímulo com hCG é um dos principais responsáveis pelo aumento da permeabilidade, levando a extravasamento do líquido intravascular para o terceiro espaço. Dentre os mediadores responsáveis por essa reação vascular destacam-se: as concentração elevadas de estradiol, de prostaglandinas, de histamina, das interleucinas (IL-6, IL-8, IL-2), do fator de crescimento endotelial vascular (VEGF), de endotelina-1, de insulina, e alterações no sistema renina-angiotensina e no sistema ovariano cinina-calicreína.

Em nosso estudo, a SHO mostrou ser muito evidente em mulheres com SOP durante a FIV. Nesse subgrupo de pacientes a taxa de fertilização foi menor e a taxa de gravidez não foi diferente quando comparada com aquelas que não desenvolveram SHO. Aboulghar et al. ${ }^{34}$ compararam 42 pacientes com SHO grave após estimulação ovariana para FIV ou ICSI, encontrando aumento do número de oócitos recrutados embora o grupo com SHO apresentasse reduzida quantidade de oócitos de boa qualidade, baixas taxas de fertilização e de implantação quando comparadas com mulheres submetidas à FIV e ICSI sem desenvolver $\mathrm{SHO}^{34}$.

Novas técnicas de reprodução assistida, como a captação de oócitos imaturos seguida da maturação in vitro destes oócitos em mulheres com SOP, têm mostrado potencial tratamento de sucesso para estas pacientes no futuro. Para isso, é necessário que seus óvulos possuam competência para maturação e desenvolvimento ${ }^{35}$. Esta conduta tem apresentado maiores benefícios que a FIV (por evitar o risco da SHO), menores custos e baixas taxas de complicações em mulheres com SOP.

Dessa forma, o sucesso da FIV está comprometido em mulheres com SOP por apresentarem recrutamento de maior número de folículos com diâmetros reduzidos, taxa reduzida de fertilização e elevada taxa de SHO.

\section{Abstract}

Purpose: to evaluate the results of ovulation hyperinduction followed by in vitro fertilization (IVF) in women with polycystic ovary syndrome (POS), as compared to normal cycle women.

Methods: a controlled retrospective study conducted on 36 women with POS (POS group) and on 44 women with infertility due to mild male factor (control group), submitted to IVF from 1997 to 2003. Subject ages ranged from 18 to 36 years. Ovulation hyperinduction was obtained with recombinant follicle-stimulating hormone and a gonadotrophin-releasing hormone agonist. The analyzed variables were the follicles with a mean diameter of 14 to 17 
$\mathrm{mm}$ and the follicles with diameters of $18 \mathrm{~mm}$ or above on the day of human chorionic gonadotrophin administration, percentage of follicles $\geq 18 \mathrm{~mm}$, the number of retrieved oocytes, fertilization rate, cleavage rate, incidence of ovarian hyperstimulation syndrome (OHS), clinical pregnancy rate, and abortion rate. The variables were analyzed by the unpaired t test, Fisher exact test and Mann-Whitney test, with level of significance set at $p<0.05$.

Results: the POS group presented a larger number of retrieved follicles, most of them measuring 14 to $17 \mathrm{~mm}$ in diameter, compared to the control group (64.8 vs 53.9\%), a lower fertilization rate (59.43 vs $79.57 \%$ ) and a higher incidence of OHS (38.9 vs 9.1\%). The number of retrieved oocytes, cleavage rates, pregnancy rates per embryo transfer, abortion rates and live born rates did not differ between groups.

Conclusion: the success of IVF is impaired in women with $P O S$ due to their larger number of retrieved follicles of reduced diameter, reduced fertilization rate and high $O H S$ rates.

KEYWORDS: Polycystic ovary syndrome. In vitro fertilization. Ovulation induction. Fertilization rate. Ovarian hyperstimulation syndrome.

\section{Agradecimentos}

À equipe do Laboratório de Reprodução Humana: Maria Cristina Picinato Medeiros de Araújo, Marilda Hatsumi Yamada Dantas, Maria Aparecida Carneiro Vasconcelos, Sandra Aparecida Cavichiollo Viana, Maria Auxiliadora Rosa, Maria Albina Verceze Bortolieiro, Ilza Alves Resende Mazzocato, Luis Alberto Manetta, Stael Porto Leite e Rodrigo Alves Ferreira.

\section{Referências}

1. Balen AH, Conway GS, Kaltsas G, et al. Polycystic ovary syndrome: the spectrum of the disorder in 1741 patients. Hum Reprod 1995; 10:2107-11.

2. Rotterdam ESHRE/ASRM-Sponsered PCOS Consensus Workshop Group. Revised 2003 consensus on diagnostic criteria and long-term health risks related to polycystic ovary syndrome. Fertil Steril 2004; 81:19-25.

3. Hull MG. Epidemiology of infertility and polycystic ovarian disease: endocrinological and demographic studies. Gynecol Endocrinol 1987; 1:235-45.

4. Franks S. Polycystic ovary syndrome. N Engl J Med 1995; 333:853-61.
5. Gilling-Smith C, Willis DS, Bear RW, Franks S. Hypersecretion of androstenedione by isolated thecal cells from polycystic ovaries. J Clin Endocrinol Metab 1994; 79:1158-65.

6. The ESHRE Capri Workshop. European Society for Human Reproduction and Embryology. Female infertility: treatment options for complicated cases. Hum Reprod 1997; 12:1191-6.

7. Golan A, Ron-El R, Herman A, Soffer Y, Weinraub $Z$, Caspi E. Ovarian hyperstimulation syndrome: an update review. Obstet Gynecol Surv 1989; 44:430-40.

8. Solomon CG. The epidemiology of polycystic ovary syndrome. Prevalence and associated disease risks. Endocrinol Metab Clin North Am 1999; 28:247-63.

9. Balen A, Michelmore K. What is polycystic ovary syndrome? Hum Reprod 2002; 17:22 19-27.

10.Homburg R, Berkowitz D, Levy T, Feldberg D, Ashkenazi, Ben-Rafael $Z$. In vitro fertilization and embryo transfer for the treatment of infertility associated with polycystic ovary syndrome. Fertil Steril 1993; 60:858-63.

11.MacDougall MJ, Tan SL, Balen A, Jacobs HS. A controlled study comparing patients with and without polycystic ovaries undergoing in-vitro fertilization. Hum Reprod 1993; 8:233-7.

12.Dor J, Shulman A, Levran D, Ben-Rafael Z, Rudak $\mathrm{E}$, Mashiach S. The treatment of patients with polycystic ovarian syndrome by in-vitro fertilization and embryo transfer: a comparison of results with those of patients with tubal infertility. Hum Reprod 1990; 5:816-8.

13. Franks S, Roberts LR, Hardy K. Gonadotropin regimens and oocyte quality in women with polycystic ovary syndrome. Reprod Biomed Online 2003; 6:181-4.

14. Cano F, Garcia-Velasco JA, Millet A, Remohi J, Simon C, Pellicer A. Oocyte quality in polycystic ovaries revisited: identification of a particular subgroup of women. J Assist Reprod Genet 1997; 14:254-61.

15. Ferriman D, Gallwey JD. Clinical assessment of body hair growth in women. J Clin Endocrinol Metab 1961; 21:1440-7.

16.World Health Organization. Laboratory manual for the examination of human semen and sperm-cervial mucus interaction. $1^{\text {st }}$ ed. Cambridge: Cambridge University Press; 1994.

17.Kruger TF, Menkveld R, Stander FS, et al. Sperm morphologic features as a prognostic factor in in vitro fertilization. Fertil Steril 1986; 46:1118-23.

18.Zollner U, Schleyer M, Steck T. Evaluation of a cutoff value for normal sperm morphology using strict criteria to predict fertilization after conventional invitro fertilization and embryo transfer in asthenozoospermia. Hum Reprod 1996; 11:2155-61. 
19.Frisdtrom M, Sjoblom P, Granberg M, Hillensjo T. A cost comparison of infertility treatment for clomiphene resistant polycystic ovary syndrome. Acta Obstet Gynecol Scand 1999; 78:212-6.

20.Homburg R, Levy T, Berkovitz D, et al. Gonadotropinreleasing hormone agonist reduces the miscarriage rate for pregnancies achieved in women with polycystic ovarian syndrome. Fertil Steril 1993; 59:527-31.

21. Quenby SM, Farquharson RG. Predicting recurring miscarriage: what is important? Obstet Gynecol 1993; 82:132-8.

22.Tarlatzis BC, Grimbizis G, Pournaropoulos F, et al. The prognostic value of basal luteinizing hormone: follicle-stimulating hormone ratio in the treatment of patients with polycystic ovarian syndrome by assisted reproduction techniques. Hum Reprod 1995; 10:2545-9.

23. Balen AH, Tan SL, MacDougall J, Jacobs HS. Miscarriage rates following in-vitro fertilization are increased in women with polycystic ovaries and reduced by pituitary desensitization with buserelin. Hum Reprod 1993; 8:959-64.

24.Wang JX, Davies MJ, Norman RJ. Polycystic ovarian syndrome and the risk of spontaneous abortion following assisted reproductive technology. Hum Reprod 2001; 16:2606-9.

25.Kodama H, Fukuda J, Karube H, Matsui T, Shimizu Y, Tanaka T. High incidence of embryo transfer cancellations in patients with polycystic ovarian syndrome. Hum Reprod 1995; 10:1962-7.

26.Smitz J, Camus M, Devroey P, Erard P, Wisanto A, Van Steirteghem AC. Incidence of severe hyperstimulation syndrome after GnRH agonist/ HMG superovulation for in-vitro fertilization. Hum Reprod 1990; 5:933-7.
27.Brinsden PR, Wada I, Tan SL, Balen A, Jacobs HS. Diagnosis, prevention and management of ovarian hyperstimulation syndrome. Br J Obstet Gynaecol 1995; 102:767-72.

28.Marci R, Senn A, Dessole S, et al. A low-dose stimulation protocol using highly purified folliclestimulating hormone can lead to high pregnancy rates in in vitro fertilization patients with polycystic ovaries who are at risk of a high ovarian response to gonadotropins. Fertil Steril 2001; 75:1131-5.

29.Buyalos RP, Lee CT. Polycystic ovary syndrome: pathophysiology and outcome with in vitro fertilization. Fertil Steril 1996; 65:1-10.

30.Tarlatzis BC, Grimbizis G. The significance of high follicular-phase luteinizing hormone levels in treatment of women with polycystic ovary syndrome by in vitro fertilization. J Assist Reprod Genet 1997; 14:1-4.

31.Plachot M, Belaisch-Allart J, Mayenga JM, et al. Oocyte and embryo quality in polycystic ovary syndrome. Gynecol Obstet Fertil 2003; 31:350-4.

32.Engmann L, Maconochie N, Sladkevicius P, Bekir J, Campbell S, Tan SL. The outcome of in-vitro fertilization treatment in women with sonographic evidence of polycystic ovarian morphology. Hum Reprod 1999; 14:167-71.

33.Balen A. Ovulation induction for polycystic ovary syndrome. Hum Fertil (Camb) 2000; 3:106-11.

34.Aboulghar M, Mansour RT, Serour GI, Ramzy AM, Amin YM. Oocyte quality in patients with severe ovarian hyperstimulation syndrome. Fertil Steril 1997; 68:1017-21.

35.Trounson A, Wood C, Kausche A. In vitro maturation and the fertilization and developmental competence of oocytes recovered from untreated polycystic ovarian patients. Fertil Steril 1994; 62:353-62.

Recebido em: 10/8/04 Aceito com modificações em: 4/10/04 